Скопје, Македонија

\title{
GAUSS-STEFFENSEN TYPE INEQUALITIES
}

\author{
JOSIP PEČARIĆ AND KSENIJA SMOLJAK KALAMIR
}

\begin{abstract}
In this paper we extend Gauss-Steffensen's inequality to the class of functions that are "convex at point $c$ ". As a consequence we obtain GaussSteffensen type inequality for convex functions. Further, we produce linear functional which generates exponential convexity and Stolarsky type means.
\end{abstract}

\section{INTRODUCTION}

In [3] Gauss mentioned the following inequality:

Theorem 1. If $f$ is a nonnegative and nonincreasing function and $k>0$ then

$$
\int_{k}^{\infty} f(x) d x \leq \frac{4}{9 k^{2}} \int_{0}^{\infty} x^{2} f(x) d x
$$

The well-known Steffensen inequality reads (see [10]):

Theorem 2. Suppose that $f$ is nonincreasing and $g$ is integrable on $[a, b]$ with $0 \leq g \leq 1$ and $\lambda=\int_{a}^{b} g(t) d t$. Then we have

$$
\int_{b-\lambda}^{b} f(t) d t \leq \int_{a}^{b} f(t) g(t) d t \leq \int_{a}^{a+\lambda} f(t) d t .
$$

The inequalities are reversed for $f$ nondecreasing.

In [5] Pečarić proved the following result.

Theorem 3. Let $G:[a, b] \rightarrow \mathbb{R}$ be an increasing function and let $f: I \rightarrow \mathbb{R}$ be $a$ nonincreasing function ( $I$ is an interval from $\mathbb{R}$ such that $a, b, G(a), G(b) \in I$ ). If $G(x) \geq x$ then

$$
\int_{G(a)}^{G(b)} f(x) d x \leq \int_{a}^{b} f(x) G^{\prime}(x) d x .
$$

If $G(x) \leq x$, the reverse inequality in (1.1) is valid.

If $f$ is a nondecreasing function and $G(x) \geq x$ then the inequality (1.1) is reversed.

2010 Mathematics Subject Classification. Primary: 26D15, Secondary: 26A51.

Key words and phrases. Gauss' inequality, Steffensen's inequality, convex function, exponential convexity, Stolarsky type means. 
Inequality (1.1) is usually called Gauss-Steffensen's inequality. As pointed out in [9] Gauss-Steffensen's inequality includes as special cases three famous inequalities: Volkov's, Steffensen's and Ostrowski's inequality.

The aim of this paper is to obtain Gauss-Steffensen type inequalities by extending Gauss-Steffensen's inequality to the class of convex functions. Further, we give an application of obtained inequalities to Stolarsky type means.

Throughout the paper we assume that $I$ is an interval from $\mathbb{R}$ containing $a, b, G(a)$ and $G(b)$.

First, let us recall some notions; log denotes the natural logarithm function and by $I^{\circ}$ we denote the interior of interval $I$.

\section{MAin RESUlts}

In [8] Pečarić and Smoljak introduced a new class of functions that extends the class of convex functions. Let us recall the definition.

Definition 2.1: Let $f: I \rightarrow \mathbb{R}$ and $c \in I^{\circ}$. We say that $f$ belongs to class $\mathcal{M}_{1}^{c}(I)$ (resp. $\left.\mathcal{M}_{2}^{c}(I)\right)$ if there exists a constant $A$ such that the function $F(x)=f(x)-A x$ is nonincreasing (resp. nondecreasing) on $I \cap(-\infty, c]$ and nondecreasing (resp. nonincreasing) on $I \cap[c, \infty)$.

We can describe the property from the previous definition as "convexity (concavity) at point $c$ ".

Remark 2.1: If $f \in \mathcal{M}_{1}^{c}(I)$ or $f \in \mathcal{M}_{2}^{c}(I)$ and $f^{\prime}(c)$ exists, then $f^{\prime}(c)=A$.

Let us show this for $f \in \mathcal{M}_{1}^{c}(I)$. Since $F$ is nonincreasing on $I \cap(-\infty, c]$ and nondecreasing on $I \cap[c, \infty)$ for every distinct points $x_{1}, x_{2} \in I \cap(-\infty, c]$ and $y_{1}, y_{2} \in I \cap[c, \infty)$ we have

$$
\left[x_{1}, x_{2} ; F\right]=\left[x_{1}, x_{2} ; f\right]-A \leq 0 \leq\left[y_{1}, y_{2} ; f\right]-A=\left[y_{1}, y_{2} ; F\right] .
$$

Therefore, since $f_{-}^{\prime}(c)$ and $f_{+}^{\prime}(c)$ exist, letting $x_{1}=y_{1}=c, x_{2} \nearrow c$ and $y_{2} \searrow c$ we get

$$
f_{-}^{\prime}(c) \leq A \leq f_{+}^{\prime}(c) .
$$

In the following theorem we recall the connection between the class of functions $\mathcal{M}_{1}^{c}[a, b]$ and the class of convex functions proved in [8].

Theorem 4. The function $f: I \rightarrow \mathbb{R}$ is convex (concave) on $I$ if and only if it is convex (concave) at every $c \in I^{\circ}$.

In the following theorem we obtain Gauss-Steffensen type inequality for class of functions that are "convex at point $c$ ".

Theorem 5. Let $G:[a, b] \rightarrow \mathbb{R}$ be an increasing function such that $G(x) \geq x$ and let $c \in(a, b)$. If $f \in \mathcal{M}_{1}^{c}(I)$ and

$$
\begin{aligned}
& \int_{a}^{c} G(t) d t-\int_{c}^{b} G(t) d t=2 c G(c)-a G(a)-b G(b)+\frac{G^{2}(b)+G^{2}(a)-2 G^{2}(c)}{2}, \\
& \text { then } \\
& \qquad \int_{a}^{c} f(t) G^{\prime}(t) d t-\int_{c}^{b} f(t) G^{\prime}(t) d t \geq \int_{G(a)}^{G(c)} f(t) d t-\int_{G(c)}^{G(b)} f(t) d t
\end{aligned}
$$


holds.

If $f \in \mathcal{M}_{2}^{c}(I)$ and (2.2) holds, the inequality in (2.3) is reversed.

Proof. Let $A$ be the constant from Definition 2.1 and let $f \in \mathcal{M}_{1}^{c}(I)$. We have $c \in(a, b) \subseteq I^{\circ}$. Let us consider the function $F: I \rightarrow \mathbb{R}, F(x)=f(x)-A x$. Since $F$ is nonincreasing on $I \cap(-\infty, c]$ we can apply inequality (1.1) to the function $F$, so

$$
\int_{G(a)}^{G(c)} F(t) d t \leq \int_{a}^{c} F(t) G^{\prime}(t) d t .
$$

Hence, we obtain

$$
\begin{aligned}
& 0 \leq \int_{a}^{c} F(t) G^{\prime}(t) d t-\int_{G(a)}^{G(c)} F(t) d t= \\
& =\int_{a}^{c} f(t) G^{\prime}(t) d t-\int_{G(a)}^{G(c)} f(t) d t-A\left(c G(c)-a G(a)-\int_{a}^{c} G(t) d t-\frac{G^{2}(c)-G^{2}(a)}{2}\right) .
\end{aligned}
$$

Further, the function $F$ is nondecreasing on $I \cap[c, \infty)$ so we can apply the reverse inequality (1.1), so we have

$$
\int_{c}^{b} F(t) G^{\prime}(t) d t \leq \int_{G(c)}^{G(b)} F(t) d t .
$$

Hence, we obtain

$$
\begin{aligned}
& 0 \leq \int_{G(c)}^{G(b)} F(t) d t-\int_{c}^{b} F(t) G^{\prime}(t) d t= \\
& =\int_{G(c)}^{G(b)} f(t) d t-\int_{c}^{b} f(t) G^{\prime}(t) d t-A\left(\frac{G^{2}(b)-G^{2}(c)}{2}-b G(b)+c G(c)+\int_{c}^{b} G(t) d t\right) .
\end{aligned}
$$

Now combining (2.4) and (2.5) we obtain

$$
\begin{aligned}
& \int_{a}^{c} f(t) G^{\prime}(t) d t-\int_{G(a)}^{G(c)} f(t) d t-\int_{c}^{b} f(t) G^{\prime}(t) d t+\int_{G(c)}^{G(b)} f(t) d t \geq \\
& \geq A\left(2 c G(c)-a G(a)-b G(b)-\int_{a}^{c} G(t) d t+\int_{c}^{b} G(t) d t+\frac{G^{2}(a)+G^{2}(b)-2 G^{2}(c)}{2}\right) .
\end{aligned}
$$

Now, from (2.2), we conclude that (2.3) holds.

Proof for $f \in \mathcal{M}_{2}^{c}(I)$ is similar so we omit the details.

As a consequence of previous theorem we obtain the following Gauss-Steffensen type inequality for class of convex functions.

Corollary 5.1. Let $G:[a, b] \rightarrow \mathbb{R}$ be an increasing function such that $G(x) \geq x$ and let $c \in(a, b)$. If $f: I \rightarrow \mathbb{R}$ is convex and (2.2) holds then (2.3) holds. If $f: I \rightarrow \mathbb{R}$ is concave and (2.2) holds, the inequality in (2.3) is reversed.

Proof. Since the function $f$ is convex, from Theorem 4, we have that $f \in \mathcal{M}_{1}^{c}(I)$ for every $c \in(a, b) \subseteq I^{\circ}$. Hence, we can apply Theorem 5 .

Remark 2.2: If the function $G$ in Theorem 5 and Corollary 5.1 is such that $G(x) \leq x$, then the reverse inequality in (2.3) holds. 
Remark 2.3: Condition (2.2) can be weakened. From the proof of Theorem 5 we have that for $f \in \mathcal{M}_{1}^{c}(I)$ condition (2.2) can be replaced by the weaker condition

$$
A\left(2 c G(c)-a G(a)-b G(b)-\int_{a}^{c} G(t) d t+\int_{c}^{b} G(t) d t+\frac{G^{2}(a)+G^{2}(b)-2 G^{2}(c)}{2}\right) \geq 0,
$$

where $A$ is the constant from Definition 2.1. Also, for $f \in \mathcal{M}_{2}^{c}(I)$ condition (2.2) can be replaced by condition (2.6) with the reverse inequality.

Furthermore, condition (2.2) can be further weakened if the function $f$ is monotonic. Since (2.1) holds, if $f \in \mathcal{M}_{1}^{c}(I)$ is nondecreasing or $f \in \mathcal{M}_{2}^{c}(I)$ is nonincreasing, from (2.6) we obtain that (2.2) can be weakened to

$$
\int_{a}^{c} G(t) d t-\int_{c}^{b} G(t) d t \leq 2 c G(c)-a G(a)-b G(b)+\frac{G^{2}(a)+G^{2}(b)-2 G^{2}(c)}{2} .
$$

Also, if $f \in \mathcal{M}_{1}^{c}(I)$ is nonincreasing or $f \in \mathcal{M}_{2}^{c}(I)$ is nondecreasing, (2.2) can be weakened to (2.7) with the reverse inequality.

\section{Mean VAlue theOREMS AND $n$-EXPONEnTial CONVEXity}

We begin this section with mean value theorems related to Gauss-Steffensen type inequality obtained in previous section. Let us define the following linear functional:

$$
L(f)=\int_{a}^{c} f(t) G^{\prime}(t) d t-\int_{c}^{b} f(t) G^{\prime}(t) d t-\int_{G(a)}^{G(c)} f(t) d t+\int_{G(c)}^{G(b)} f(t) d t .
$$

Remark 3.1: Under assumptions of Theorem 5 we have that $L(f) \geq 0$ for $f \in$ $\mathcal{M}_{1}^{c}(I)$. Further, under assumptions of Corollary 5.1 we have that $L(f) \geq 0$ for any convex function $f$.

First, we give the Lagrange type mean value theorem.

Theorem 6. Let $G:[a, b] \rightarrow \mathbb{R}$ be an increasing function such that $G(x) \geq x$ and let $c \in(a, b)$. Assume that (2.2) holds. Then for any $f \in C^{2}(I)$ there exists $\xi \in I$ such that

$$
L(f)=\frac{f^{\prime \prime}(\xi)}{2}\left[\int_{a}^{c} x^{2} G^{\prime}(x) d x-\int_{c}^{b} x^{2} G^{\prime}(x) d x+\frac{G^{3}(b)+G^{3}(a)-2 G^{3}(c)}{3}\right],
$$

where $L$ is defined by (3.1).

Proof. Since $f \in C^{2}(I)$ there exist

$$
m=\min _{x \in I} f^{\prime \prime}(x) \quad \text { and } \quad M=\max _{x \in I} f^{\prime \prime}(x) .
$$

The functions

$$
\Phi_{1}(x)=f(x)-\frac{m}{2} x^{2} \quad \text { and } \quad \Phi_{2}(x)=\frac{M}{2} x^{2}-f(x)
$$


are convex since $\Phi_{i}^{\prime \prime}(x) \geq 0, i=1,2$. Hence, by Remark 3.1 we have $L\left(\Phi_{i}\right) \geq 0$, $i=1,2$ and we obtain

$$
\frac{m}{2} L\left(x^{2}\right) \leq L(f) \leq \frac{M}{2} L\left(x^{2}\right),
$$

where

$$
L\left(x^{2}\right)=\int_{a}^{c} x^{2} G^{\prime}(x) d x-\int_{c}^{b} x^{2} G^{\prime}(x) d x+\frac{G^{3}(b)+G^{3}(a)-2 G^{3}(c)}{3} .
$$

Since $x^{2}$ is convex, by Remark 3.1 we have $L\left(x^{2}\right) \geq 0$.

If $L\left(x^{2}\right)=0$, then (3.3) implies $L(f)=0$ and (3.2) holds for every $\xi \in I$. Otherwise, dividing (3.3) by $L\left(x^{2}\right) / 2>0$ we get

$$
m \leq \frac{2 L(f)}{L\left(x^{2}\right)} \leq M,
$$

so continuinity of $f^{\prime \prime}$ ensures existence of $\xi \in I$ satisfying (3.2).

We continue with the Cauchy type mean value theorem.

Theorem 7. Let $G:[a, b] \rightarrow \mathbb{R}$ be an increasing function such that $G(x) \geq x$ and let $c \in(a, b)$. Assume that (2.2) holds. Then for any $f, h \in C^{2}(I)$ such that $h^{\prime \prime}(x) \neq 0$ for every $x \in I$, there exists $\xi \in I$ such that

$$
\frac{L(f)}{L(h)}=\frac{f^{\prime \prime}(\xi)}{h^{\prime \prime}(\xi)}
$$

holds, where $L$ is defined by (3.1).

Proof. Let us define $\Phi \in C^{2}(I)$ by $\Phi(x)=L(h) f(x)-L(f) h(x)$. Due to linearity of $L$ we have $L(\Phi)=0$. Now, by Theorem 6 there exist $\xi, \eta \in I$ such that

$$
\begin{aligned}
& 0=L(\Phi)=\frac{\Phi^{\prime \prime}(\xi)}{2} L\left(x^{2}\right) \\
& 0 \neq L(h)=\frac{h^{\prime \prime}(\eta)}{2} L\left(x^{2}\right) .
\end{aligned}
$$

Therefore, $L\left(x^{2}\right) \neq 0$ and

$$
0=\Phi^{\prime \prime}(\xi)=L(h) f^{\prime \prime}(\xi)-L(f) h^{\prime \prime}(\xi),
$$

which gives the claim of the theorem.

Let us recall definition and some results on exponential and $n$-exponential convexity, for more details see [1], [2], [4] and [6].

Definition 3.1: A function $\psi: J \rightarrow \mathbb{R}$ is $n$-exponentially convex in the Jensen sense on $J$ if

$$
\sum_{i, j=1}^{n} \xi_{i} \xi_{j} \psi\left(\frac{x_{i}+x_{j}}{2}\right) \geq 0
$$

holds for all choices $\xi_{1}, \ldots, \xi_{n} \in \mathbb{R}$ and all choices $x_{1}, \ldots, x_{n} \in J$.

A function $\psi: J \rightarrow \mathbb{R}$ is n-exponentially convex on $J$ if it is $n$-exponentially convex in the Jensen sense and continuous on $J$. 
Remark 3.2: It is clear from the definition that 1-exponentially convex functions in the Jensen sense are in fact nonnegative functions.

Also, $n$-exponentially convex functions in the Jensen sense are $k$-exponentially convex in the Jensen sense for every $k \leq n, k \in \mathbb{N}$.

Definition 3.2: A function $\psi: J \rightarrow \mathbb{R}$ is exponentially convex in the Jensen sense on $J$ if it is $n$-exponentially convex in the Jensen sense on $J$ for every $n \in \mathbb{N}$.

A function $\psi: J \rightarrow \mathbb{R}$ is exponentially convex on $J$ if it is exponentially convex in the Jensen sense and continuous on $J$.

Remark 3.3: A function $\psi: J \rightarrow \mathbb{R}$ is log-convex in the Jensen sense, i.e.

$$
\psi\left(\frac{x+y}{2}\right)^{2} \leq \psi(x) \psi(y), \quad \text { for all } x, y \in J,
$$

if and only if

$$
\alpha^{2} \psi(x)+2 \alpha \beta \psi\left(\frac{x+y}{2}\right)+\beta^{2} \psi(y) \geq 0
$$

holds for every $\alpha, \beta \in \mathbb{R}$ and $x, y \in J$, i.e., if and only if $\psi$ is 2-exponentially convex in the Jensen sense. By induction from (3.4) we have

$$
\psi\left(\frac{1}{2^{k}} x+\left(1-\frac{1}{2^{k}}\right) y\right) \leq \psi(x)^{\frac{1}{2^{k}}} \psi(y)^{1-\frac{1}{2^{k}}}
$$

Therefore, if $\psi$ is continuous and $\psi(x)=0$ for some $x \in J$, then from the last inequality and nonnegativity of $\psi$ (see Remark 3.2) we get

$$
\psi(y)=\lim _{k \rightarrow \infty} \psi\left(\frac{1}{2^{k}} x+\left(1-\frac{1}{2^{k}}\right) y\right)=0 \quad \text { for all } y \in J
$$

Hence, 2-exponentially convex function is either identically equal to zero or it is strictly positive and log-convex.

The following lemma is equivalent to the definition of convex functions (see [7]).

Lemma 1. A function $\psi: J \rightarrow \mathbb{R}$ is convex if and only if the inequality

$$
\left(x_{3}-x_{2}\right) \psi\left(x_{1}\right)+\left(x_{1}-x_{3}\right) \psi\left(x_{2}\right)+\left(x_{2}-x_{1}\right) \psi\left(x_{3}\right) \geq 0
$$

holds for all $x_{1}, x_{2}, x_{3} \in J$ such that $x_{1}<x_{2}<x_{3}$.

We also use the following result (see [7]).

Proposition 3.1. If $f$ is a convex function on $J$ and if $x_{1} \leq y_{1}, x_{2} \leq y_{2}, x_{1} \neq x_{2}$, $y_{1} \neq y_{2}$, then the following inequality holds

$$
\frac{f\left(x_{2}\right)-f\left(x_{1}\right)}{x_{2}-x_{1}} \leq \frac{f\left(y_{2}\right)-f\left(y_{1}\right)}{y_{2}-y_{1}} .
$$

If the function $f$ is concave, the inequality is reversed.

Definition 3.3: The second order devided difference of a function $f: J \rightarrow \mathbb{R}, J$ is an interval in $\mathbb{R}$, at mutually different points $x_{0}, x_{1}, x_{2} \in J$ is defined recursively by

$$
\left[x_{i} ; f\right]=f\left(x_{i}\right), \quad i=0,1,2
$$




$$
\begin{gathered}
{\left[x_{i}, x_{i+1} ; f\right]=\frac{f\left(x_{i+1}\right)-f\left(x_{i}\right)}{x_{i+1}-x_{i}}, \quad i=0,1} \\
{\left[x_{0}, x_{1}, x_{2} ; f\right]=\frac{\left[x_{1}, x_{2} ; f\right]-\left[x_{0}, x_{1} ; f\right]}{x_{2}-x_{0}} .}
\end{gathered}
$$

Remark 3.4: The value $\left[x_{0}, x_{1}, x_{2} ; f\right]$ is independent of the order of the points $x_{0}, x_{1}$ and $x_{2}$. This definition may be extended to include the case in which some or all the points coincide. Taking the limit $x_{1} \rightarrow x_{0}$ in (3.5), we get

$$
\lim _{x_{1} \rightarrow x_{0}}\left[x_{0}, x_{1}, x_{2} ; f\right]=\left[x_{0}, x_{0}, x_{2} ; f\right]=\frac{f\left(x_{2}\right)-f\left(x_{0}\right)-f^{\prime}\left(x_{0}\right)\left(x_{2}-x_{0}\right)}{\left(x_{2}-x_{0}\right)^{2}}, \quad x_{2} \neq x_{0}
$$

provided that $f^{\prime}$ exists, and furthermore, taking the limits $x_{i} \rightarrow x_{0}, i=1,2$ in (3.5), we get

$$
\lim _{x_{2} \rightarrow x_{1}} \lim _{x_{1} \rightarrow x_{0}}\left[x_{0}, x_{1}, x_{2} ; f\right]=\left[x_{0}, x_{0}, x_{0} ; f\right]=\frac{f^{\prime \prime}\left(x_{0}\right)}{2}
$$

provided that $f^{\prime \prime}$ exists.

In the following theorem we show $n$-exponential convexity of functional $L$. Similar result was proved in [8] so we omit the proof. In the sequel $J$ and $K$ denote intervals in $\mathbb{R}$.

Theorem 8. Let $\Omega=\left\{f_{p}: J \rightarrow \mathbb{R} \mid p \in K\right\}$ be a family of functions such that for every mutually different points $x_{0}, x_{1}, x_{2} \in J$ the mapping $p \mapsto\left[x_{0}, x_{1}, x_{2} ; f_{p}\right]$ is $n$-exponentially convex in the Jensen sense on $K$. Let $L$ be linear functional defined by (3.1). Then the mapping $p \mapsto L\left(f_{p}\right)$ is $n$-exponentially convex in the Jensen sense on $K$.

If the mapping $p \mapsto L\left(f_{p}\right)$ is continuous on $K$, then it is $n$-exponentially convex on $K$.

If the assumptions of Theorem 8 hold for all $n \in \mathbb{N}$, then we have the following corollary.

Corollary 8.1. Let $\Omega=\left\{f_{p}: J \rightarrow \mathbb{R} \mid p \in K\right\}$ be a family of functions such that for every mutually different points $x_{0}, x_{1}, x_{2} \in J$ the mapping $p \mapsto\left[x_{0}, x_{1}, x_{2} ; f_{p}\right]$ is exponentially convex in the Jensen sense on $K$. Let $L$ be linear functional defined by (3.1). Then the mapping $p \mapsto L\left(f_{p}\right)$ is exponentially convex in the Jensen sense on $K$.

If the mapping $p \mapsto L\left(f_{p}\right)$ is continuous on $K$, then it is exponentially convex on $K$.

We continue with the result which is useful for the application to Stolarsky type means. Again, similar result was obtained in [8] so we recall it without the proof.

Corollary 8.2. Let $\Omega=\left\{f_{p}: J \rightarrow \mathbb{R} \mid p \in K\right\}$ be a family of functions such that for every mutually different points $x_{0}, x_{1}, x_{2} \in J$ the mapping $p \mapsto\left[x_{0}, x_{1}, x_{2} ; f_{p}\right]$ is 2-exponentially convex in the Jensen sense on $K$. Let $L$ be linear functional defined by (3.1). Then the following statements hold: 
(i) If the mapping $p \mapsto L\left(f_{p}\right)$ is continuous on $K$, then for $r, s, t \in K$, such that $r<s<t$, we have

$$
\left[L\left(f_{s}\right)\right]^{t-r} \leq\left[L\left(f_{r}\right)\right]^{t-s}\left[L\left(f_{t}\right)\right]^{s-r} .
$$

(ii) If the mapping $p \mapsto L\left(f_{p}\right)$ is strictly positive and differentiable on $K$, then for every $p, q, u, v \in K$ such that $p \leq u$ and $q \leq v$ we have

$$
\mu_{p, q}(L, \Omega) \leq \mu_{u, v}(L, \Omega)
$$

where

$$
\mu_{p, q}(L, \Omega)= \begin{cases}\left(\frac{L\left(f_{p}\right)}{L\left(f_{q}\right)}\right)^{\frac{1}{p-q}}, & p \neq q, \\ \exp \left(\frac{\frac{d}{d p} L\left(f_{p}\right)}{L\left(f_{p}\right)}\right), & p=q .\end{cases}
$$

Remark 3.5: Results from Theorem 8, Corollaries 8.1 and 8.2 still hold when two of the points $x_{0}, x_{1}, x_{2} \in J$ coincide, say $x_{1}=x_{0}$, for a family of differentiable functions $f_{p}$ such that the function $p \rightarrow\left[x_{0}, x_{1}, x_{2} ; f_{p}\right]$ is $n$-exponentially convex in the Jensen sense (exponentially convex in the Jensen sense, log-convex in the Jensen sense), and furthermore, they still hold when all three points coincide for a family of twice differentiable functions with the same property. The proofs are obtained by recalling Remark 3.4 and suitable characterization of convexity.

We continue with some families of functions for which we use Corollaries 8.1 and 8.2 to construct exponentially convex functions and Stolarsky type means related to Gauss-Steffensen type inequality.

\section{Example 3.1: Let}

$$
\Upsilon_{1}=\left\{f_{p}: \mathbb{R} \rightarrow[0, \infty) \mid p \in \mathbb{R}\right\}
$$

be a family of functions defined by

$$
f_{p}(x)= \begin{cases}\frac{e^{p x}}{p^{2}}, & p \neq 0 \\ \frac{x^{2}}{2}, & p=0\end{cases}
$$

For every $p \in \mathbb{R}$ we have that $f_{p}$ is a convex function on $\mathbb{R}$ since $\frac{d^{2}}{d x^{2}} f_{p}(x)=e^{p x}>0$. Furthermore, $p \mapsto \frac{d^{2}}{d x^{2}} f_{p}(x)$ is exponentially convex by definition. Similar as in proof of Theorem 8 we conclude that $p \mapsto\left[x_{0}, x_{1}, x_{2} ; f_{p}\right]$ is exponentially convex (and so exponentially convex in the Jensen sense). Using Corollary 8.1 we obtain that $p \mapsto L\left(f_{p}\right)$ is exponentially convex in the Jensen sense. It is easy to verify that this mapping is continuous, so it is exponentially convex. For this family of functions, from Corollary 8.2 we have the following:

* for $p \neq q, p, q \neq 0$ :

$$
\mu_{p, q}\left(L, \Upsilon_{1}\right)=\left(\frac{q^{2}}{p^{2}} \frac{\int_{a}^{c} e^{p x} G^{\prime}(x) d x-\int_{c}^{b} e^{p x} G^{\prime}(x) d x+\frac{e^{p G(b)}+e^{p G(a)}-2 e^{p G(c)}}{p}}{\int_{a}^{c} e^{q x} G^{\prime}(x) d x-\int_{c}^{b} e^{q x} G^{\prime}(x) d x+\frac{e^{q G(b)}+e^{q G(a)}-2 e^{q G(c)}}{q}}\right)^{\frac{1}{p-q}}
$$


* for $p \neq q, q=0$ (or $p=0$ ):

$$
\begin{aligned}
& \mu_{p, 0}\left(L, \Upsilon_{1}\right)=\left(\frac{2}{p^{2}} \frac{\int_{a}^{c} e^{p x} G^{\prime}(x) d x-\int_{c}^{b} e^{p x} G^{\prime}(x) d x+\frac{e^{p G(b)}+e^{p G(a)}-2 e^{p G(c)}}{p}}{\int_{a}^{c} x^{2} G^{\prime}(x) d x-\int_{c}^{b} x^{2} G^{\prime}(x) d x+\frac{G^{3}(a)+G^{3}(b)-2 G^{3}(c)}{3}}\right)^{\frac{1}{p}} \\
& \quad=\mu_{0, p}\left(L, \Upsilon_{1}\right) \\
& * \text { for } p=q \neq 0: \\
& \mu_{p, p}\left(L, \Upsilon_{1}\right)= \\
& \exp \left(\frac{\int_{a}^{c} x e^{p x} G^{\prime}(x) d x-\int_{c}^{b} x e^{p x} G^{\prime}(x) d x+\frac{e^{p G(b)}(p G(b)-1)+e^{p G(a)}(p G(a)-1)-2 e^{p G(c)}(p G(c)-1)}{p^{2}}}{\int_{a}^{c} e^{p x} G^{\prime}(x) d x-\int_{c}^{b} e^{p x} G^{\prime}(x) d x+\frac{e^{p G(b)}+e^{p G(a)}-2 e^{p G(c)}}{p}}-\frac{2}{p}\right) \\
& \quad \text { for } p=q=0: \\
& \mu_{0,0}\left(L, \Upsilon_{1}\right)=\exp \left(\frac{1}{3} \frac{\int_{a}^{c} x^{3} G^{\prime}(x) d x-\int_{c}^{b} x^{3} G^{\prime}(x) d x+\frac{G^{4}(b)+G^{4}(a)-2 G^{4}(c)}{4}}{\int_{a}^{c} x^{2} G^{\prime}(x) d x-\int_{c}^{b} x^{2} G^{\prime}(x) d x+\frac{G^{3}(b)+G^{3}(a)-2 G^{3}(c)}{3}}\right) .
\end{aligned}
$$

Applying Theorem 7 on functions $f_{p}, f_{q} \in \Upsilon_{1}$ and functional $L$ it follows that

$$
M_{p, q}\left(L, \Upsilon_{1}\right)=\log \mu_{p, q}\left(L, \Upsilon_{1}\right)
$$

satisfies $\min I \leq M_{p, q}\left(L, \Upsilon_{1}\right) \leq \max I$. So $M_{p, q}\left(L, \Upsilon_{1}\right)$ is a monotonic mean by (3.6).

Example 3.2: Let

$$
\Upsilon_{2}=\left\{h_{p}:(0, \infty) \rightarrow \mathbb{R} \mid p \in \mathbb{R}\right\}
$$

be a family of functions defined by

$$
h_{p}(x)= \begin{cases}\frac{x^{p}}{p(p-1)}, & p \neq 0,1 \\ -\log x, & p=0 \\ x \log x, & p=1\end{cases}
$$

We have that $h_{p}$ is a convex function on $\mathbb{R}^{+}$since $\frac{d^{2}}{d x^{2}} h_{p}(x)=x^{p-2}>0$ for $x>0$. Furthermore, $p \mapsto \frac{d^{2}}{d x^{2}} h_{p}(x)$ is exponentially convex by definition. Smiliar as in Example 3.1 we obtain that $p \mapsto L\left(h_{p}\right)$ is exponentially convex in the Jensen sense. It is easy to verify that this mapping is continuous, so it is exponentially convex. Hence, for this family of functions, from Corollary 8.2 we have that $\mu_{p, q}\left(L, \Upsilon_{2}\right)$ is given by

$$
\mu_{p, q}\left(L, \Upsilon_{2}\right)= \begin{cases}\left(\frac{L\left(h_{p}\right)}{L\left(h_{q}\right)}\right)^{\frac{1}{p-q}}, & p \neq q ; \\ \exp \left(\frac{-L\left(h_{p} h_{0}\right)}{L\left(h_{p}\right)}-\frac{2 p-1}{p(p-1)}\right), & p=q \neq 0,1 \\ \exp \left(\frac{-L\left(h_{0}^{2}\right)}{2 L\left(h_{0}\right)}+1\right), & p=q=0 ; \\ \exp \left(\frac{-L\left(h_{0} h_{1}\right)}{2 L\left(h_{1}\right)}-1\right), & p=q=1 .\end{cases}
$$

Applying Theorem 7 on functions $h_{p}, h_{q} \in \Upsilon_{2}$ and functional $L$ we conclude that there exists $\xi \in I$ such that

$$
\xi^{p-q}=\frac{L\left(h_{p}\right)}{L\left(h_{q}\right)}
$$


Since the function $\xi \mapsto \xi^{p-q}$ is invertible for $p \neq q$ we have

$$
\min I \leq\left(\frac{L\left(h_{p}\right)}{L\left(h_{q}\right)}\right)^{\frac{1}{p-q}} \leq \max I
$$

which together with the fact that $\mu_{p, q}\left(L, \Upsilon_{2}\right)$ is continuous, symetric and monotonic (by (3.6)) shows that $\mu_{p, q}\left(L, \Upsilon_{2}\right)$ is a mean.

Acknowledgements. This work has been fully supported by Croatian Science Foundation under the project 5435.

\section{REFERENCES}

[1] N. I. Akhiezer, The classical moment problem and some related questions in analysis, Oliver and Boyd Ltd, Edinburgh and London, 1965.

[2] S. N. Bernstein, Sur les fonctions absolument monotones, Acta Math., 52 (1929), 1-66.

[3] C. F. Gauss, Theoria combinationis observationum, 1821., German transl. in Abhandlungen zur Methode der kleinsten Quadrate. Neudruck. Würzburg 1964, pp. 9 and 12.

[4] J. Jakšetić, J. Pečarić, Exponential convexity method, J. Convex Anal., 20(1) (2013), 181-197.

[5] J.E. Pečarić, Connections among some inequalities of Gauss, Steffensen and Ostrowski, Southeast Asian Bull. Math., 13(2) (1989), 89-91.

[6] J. Pečarić, J. Perić, Improvements of the Giaccardi and the Petrović inequality and related Stolarsky type means, An. Univ. Craiova Ser. Mat. Inform., 39(1) (2012), 65-75.

[7] J.E. Pečarić, F. Proschan, Y.L. Tong, Convex functions, partial orderings and statistical applications, Mathematics in Science and Engineering, 187, Academic Press Inc., Boston, 1992.

[8] J. Pečarić, K. Smoljak, Steffensen type inequalities involving convex functions, Math. Inequal. Appl., 18(1) (2015), 363-378.

[9] J. Pečarić, K. Smoljak Kalamir, S. Varošanec, Steffensen's and related inequalities (A comprehensive survey and recent advances), Monograhps in inequalities 7, Element, Zagreb, 2014.

[10] J.F. Steffensen, On certain inequalities between mean values and their application to actuarial problems, Skand. Aktuarietids. (1918), 82-97.

Faculty of Textile Technology, University of Zagreb, Prilaz baruna Filipovića 28A, 10000 Zagreb, Croatia

E-mail address: pecaric@element.hr

Faculty of Textile Technology, University of Zagreb, Prilaz baruna Filipovića 28A, 10000 Zagreb, Croatia

E-mail address: ksmoljak@ttf.hr 